\title{
Fusion of the Genes WWTRI and FOSB in Pseudomyogenic Hemangioendothelioma
}

\author{
IOANNIS PANAGOPOULOS ${ }^{1}$, INGVILD LOBMAIER $^{2}$, LUDMILA GORUNOVA $^{1}$ and SVERRE HEIM ${ }^{1,3}$ \\ ${ }^{1}$ Section for Cancer Cytogenetics, Institute for Cancer Genetics and Informatics, \\ The Norwegian Radium Hospital, Oslo University Hospital, Oslo, Norway; \\ ${ }^{2}$ Department of Pathology, the Norwegian Radium Hospital, Oslo University Hospital, Oslo, Norway; \\ ${ }^{3}$ Institute of Clinical Medicine, Faculty of Medicine, University of Oslo, Oslo, Norway
}

\begin{abstract}
Background/Aim: Pseudomyogenic hemangioendothelioma is a rare endothelial tumor. Previous genetic investigations have shown that the tumors carry either a SERPINE1-FOSB or an ACTB-FOSB fusion gene. The aim of the study was to identify FOSB fusions linked with pseudomyogenic hemangioendothelioma. Materials and Methods: RNA sequencing, reverse transcription polymerase chain reaction (RT-PCR) and Sanger sequencing analyses were performed on a pseudomyogenic hemangioendothelioma. Results: An in-frame fusion was found between exon 4 of WWTR1 from $3 q 25$ and exon 2 of FOSB from 19q13. The fusion gene not only places FOSB under the control of the WWTR1 promoter, but is predicted to encode a chimeric WWTR1-FOSB transcription factor. Conclusion: FOSB may be fused with SERPINE1, ACTB, or WWTR1 in pseudomyogenic hemangioendotheliomas. The resulting overexpression of FOSB fusion is a potentially useful marker that could be helpful in the diagnosis of these tumors.
\end{abstract}

Pseudomyogenic hemangioendothelioma is a rare endothelial neoplasm that is multifocal in two-thirds of cases and often occurs within a limited region $(1,2)$. It is more frequently found in young males and primarily in soft tissue, with only $20 \%$ presenting with secondary manifestations in bone. About $60 \%$ of the lesions occur in the lower limbs. Half of the patients experience pain. Histologically, the tumors are

This article is freely accessible online.

Correspondence to: Ioannis Panagopoulos, Section for Cancer Cytogenetics, Institute for Cancer Genetics and Informatics, The Norwegian Radium Hospital, Oslo University Hospital, Montebello, PO Box 4954 Nydalen, NO-0424 Oslo, Norway. Tel: +47 22782362, e-mail: ioannis.panagopoulos@rr-research.no

Key Words: Pseudomyogenic hemangioendothelioma, WWTR1, FOSB, WWTR1-FOSB fusion gene, RNA sequencing. ill-defined, composed of sheets and cords of spindled cells with abundant, eosinophilic cytoplasm. The nucleus is vesicular, with small nucleoli. There are few mitotic figures and little atypia. Immunohistochemistry (IHC) shows diffuse positive expression of AE1/AE3 and ERG and, in $30 \%$ of cases, positive staining for CD31 and smooth muscle actin (SMA). The tumors are negative for Desmin, S100 and CD34 (1, 2). Cytogenetic information on pseudomyogenic hemangioendothelioma is restricted to 4 tumors all of which carried a $\mathrm{t}(7 ; 19)(\mathrm{q} 22 ; \mathrm{q} 13)$ chromosomal translocation $(3,4)$. The translocation resulted in the fusion of the SERPINE1 gene from $7 \mathrm{q} 22$ with $F O S B$ gene from $19 \mathrm{q} 13$ resulting in overexpression of $F O S B$ (4). In two recent studies, fusion of $A C T B$ from $7 \mathrm{p} 22$ with $F O S B$ was described in pseudomyogenic hemangioendotheliomas lacking SERPINE1 rearrangement $(5,6)$. Agaram et al. (5) have studied 15 pseudomyogenic hemangioendotheliomas and, by using fluorescence in situ hybridization and ARCHER FusionPlex analysis, found that seven of the tumors had an ACTB-FOSB fusion whereas eight of them had a SERPINE1-FOSB fusion. Zhu et al. have detected ACTB-FOSB fusion in two pseudomyogenic hemangioendo-theliomas using target RNA sequencing (6).

We here present another pseudomyogenic hemangioendothelioma that carried none of the above-mentioned fusions, but instead a novel WWTRI-FOSB fusion gene.

\section{Materials and Methods}

Ethics statement. The study was approved by the regional ethics committee (Regional komité for medisinsk forskningsetikk Sør-Øst, Norge, http://helseforskning etikkom.no). Written informed consent was obtained from the patient for publication of the case details. The ethics committee's approval included a review of the consent procedure. All patient information has been de-identified.

Case description. The patient was a 33-year-old woman who had a history of back pain for over 6 years. A sudden worsening of her symptoms led to radiological examination which revealed a lytic lesion in the upper lateral region of the sacral bone, presumably of 
Table I. Detection of WWTR1-FOSB fusion in the RNA sequencing data of pseudomyogenic hemangioendothelioma. The retrieved sequences from the fastq file of the RNA sequencing using the "grep" command and the search term "GAGTGCGCCGGTCTCGGGGA" which is the first 20 nt in the exon 2 of FOSB corresponding to nt 719-738 in the FOSB reference sequence with the accession number NM_006732.2.

\begin{tabular}{|c|c|}
\hline Sequence & Gene \\
\hline CTACGCCTGTGTGTGTATGTGTCACCCCGTAGGAGTGCGCCGGTCTCGGGGAAATGCCCGGTTCCTTCGTGCCCA & FOSB \\
\hline GTGTATGTGTCACCCCGTAGGAGTGCGCCGGTCTCGGGGAAATGCCCGGTTCCTTCGTGCCCACGGTCACCGCGA & $F O S B$ \\
\hline CCCGTAGGAGTGCGCCGGTCTCGGGGAAATGCCCGGTTCCTTCGTGCCCACGGTCACCGCGATCACAACCAGCCA & FOSB \\
\hline CTTTGTTTGTGTGTCTACGCCTGTGTGTGTATGTGTCACCCCGTAGGAGTGCGCCGGTCTCGGGGAAATGCCCGG & FOSB \\
\hline TGTGTCTACGCCTGTGTGTGTATGTGTCACCCCGTAGGAGTGCGCCGGTCTCGGGGAAATGCCCGGTTCCTTCGT & FOSB \\
\hline CACCCCGTAGGAGTGCGCCGGTCTCGGGGAAATGCCCGGTTCCTTCGTGCCCACGGTCACCGCGATCACAACCAG & FOSB \\
\hline CATGAGGCAGGAGTGCGCCGGTCTCGGGGAAATGCCCGGTTCCTTCGTGCCCACGGTCACCGCGATCACAACCAG & WWTR1-FOSB \\
\hline GTATGTGTCACCCCGTAGGAGTGCGCCGGTCTCGGGGAAATGCCCGGTTCCTTCGTGCCCACGGTCACCGCGATC & $F O S B$ \\
\hline TGTCACCCCGTAGGAGTGCGCCGGTCTCGGGGAAATGCCCGGTTCCTTCGTGCCCACGGTCACCGCGATCACAAC & $F O S B$ \\
\hline AGTGCGCCGGTCTCGGGGAAATGCCCGGTTCCTTCGTGCCCACGGTCACCGCGATCACAACCAGCCAGGACCTCC & $F O S B$ \\
\hline TGGAGAGAGAAAGGATTCGAATGCGCCAAGAGGAGCTCATGAGGCAGGAGTGCGCCGGTCTCGGGGAAATGCCCG & WWTR1-FOSB \\
\hline GCCTGTGTGTGTATGTGTCACCCCGTAGGAGTGCGCCGGTCTCGGGGAAATGCCCGGTTCCTTCGTGCCCACGGT & FOSB \\
\hline CTCCCAGGAGTGCGCCGGTCTCGGGGAAATGCCCGGTTCCTTCGTGCCCACGGTCACCGCGATCACAACCAGCCA & $F O S B$ \\
\hline GTGTCTTTGTTTGTGTGTCTACGCCTGTGTGTGTATGTGTCACCCCGTAGGAGTGCGCCGGTCTCGGGGAAATGC & FOSB \\
\hline GGAGCTCATGAGGCAGGAGTGCGCCGGTCTCGGGGAAATGCCCGGTTCCTTCGTGCCCACGGTCACCGCGATCAC & WWTRI-FOSB \\
\hline TGTATGTGTCACCCCGTAGGAGTGCGCCGGTCTCGGGGAAATGCCCGGTTCCTTCGTGCCCACGGTCACCGCGAT & $F O S B$ \\
\hline CGCCTGTGTGTGTATGTGTCACCCCGTAGGAGTGCGCCGGTCTCGGGGAAATGCCCGGTTCCTTCGTGCCCACGG & FOSB \\
\hline GTCACCCCGTAGGAGTGCGCCGGTCTCGGGGAAATGCCCGGTTCCTTCGTGCCCACGGTCACCGCGATCACAACC & $F O S B$ \\
\hline TGTCGGTGTCTTTGTTTGTGTGTCTACGCCTGTGTGTGTATGTGTCACCCCGTAGGAGTGCGCCGGTCTCGGGGA & $F O S B$ \\
\hline GTGTCGGTGTCTTTGTTTGTGTGTCTACGCCTGTGTGTGTATGTGTCACCCCGTAGGAGTGCGCCGGTCTCGGGG & FOSB \\
\hline TATGTGTCACCCCGTAGGAGTGCGCCGGTCTCGGGGAAATGCCCGGTTCCTTCGTGCCCACGGTCACCGCGATCA & FOSB \\
\hline AGGATTCGAATGCGCCAAGAGGAGCTCATGAGGCAGGAGTGCGCCGGTCTCGGGGAAATGCCCGGTTCCTTCGTG & WWTR1-FOSB \\
\hline GTCACCCCGTAGGAGTGCGCCGGTCTCGGGGAAATGCCCGGTTCCTTCGTGCCCACGGTCACCGCGATCACAAAC & $F O S B$ \\
\hline
\end{tabular}

a benign, fibrous nature, in addition to a prolapse in the lumbar spine. The lesion in the sacrum had presumably brought about the longstanding dumb, continuous pain. Magnetic resonance imaging (MRI) and positron emission tomography (PET) examinations showed also a second lytic lesion in the lumbar spine. Examination of an initial core needle biopsy showed a spindle cell lesion without evidence of atypia leading to a tentative diagnosis of benign fibrous histiocytoma. In a second core needle biopsy, areas with giant cells were observed. Based on the evidence that a variant of giant cell tumor was present, the patient treated with Denosumab. After initial alleviation of symptoms, the pain came back after four injections of Denosumab. A new PET scan showed minimal reduction in signal intensity and no reduction in tumor size. The treatment was discontinued and an open biopsy was performed, which again revealed a spindle cell lesion without atypia (Figure 1A-B). Immunohistochemistry (IHC) showed positivity for SMA, high molecular weight cytokeratins AE1/AE3 (Figure 1C), ERG (Figure 1D), and CD31 (Figure 1E). After consulting Professor Nielsen at Massachusetts General Hospital, the patient was diagnosed with a pseudomyogenic hemangioendothelioma. IHC for FOSB was then performed which showed strong nuclear positivity (Figure 1F).

Chromosome banding analysis. Fresh tissue from a representative area of the tumor was received and isolated cells were short-term cultured and analysed cytogenetically, as part of our diagnostic routine, as described elsewhere (7).

$R N A$ sequencing. Total RNA was extracted from frozen $\left(-80^{\circ} \mathrm{C}\right)$ tumor tissue using miRNeasy Mini Kit (Qiagen Nordic, Oslo, Norway) and one $\mu \mathrm{g}$ of total RNA was sent to the Genomics Core Facility at the
Norwegian Radium Hospital, Oslo University Hospital (http://genomics.no/oslo/) for high-throughput paired-end RNAsequencing according to the Illumina TruSeq Stranded mRNA protocol. The "grep" command was used to search the fastq files of the sequence data for $F O S B$ sequence. The principle of this approach has been described in detail elsewhere $(7,8)$. The search term was the 20nucleotide-sequence (nt) "GAGTGCGCCGGTC TCGGGGA" which is the first 20 nt in exon 2 of FOSB corresponding to nt 719-738 in the FOSB reference sequence with accession number NM_006732.2.

Reverse transcription $(R T)$ and genomic PCR analyses. One $\mu \mathrm{g}$ of total RNA was reverse-transcribed in a $20 \mu \mathrm{l}$ reaction volume using iScript Advanced cDNA Synthesis Kit for RT-qPCR according to the manufacturer's instructions (Bio-Rad, Oslo, Norway). One $\mu \mathrm{l}$ of the synthesized cDNA was used as template in subsequent PCR assays. PCR amplifications were performed in a $25 \mu \mathrm{l}$ reaction volume which contained 12.5 $\mu$ Premix Ex Taq ${ }^{\text {TM }}$ DNA Polymerase Hot Start Version (Takara Bio Europe/SAS, Saint-Germain-en-Laye, France), template ( $1 \mu \mathrm{l} \mathrm{cDNA})$, and $0.4 \mu \mathrm{M}$ of each of the forward and reverse primers. The forward primer was WWTR1-996F1: TGA GTA TGC CCA ATG CGC TGA CCA corresponding to position 996-1019 in the WWTR1 reference sequence with accession number NM_015472.4. The reverse primer was FOSB-817R1: TGG GAC TGG GCC ATG GAA GAG ATG corresponding to position 840-817 in the $F O S B$ reference sequence with accession number NM_006732.2. PCR amplifications were run on a C-1000 Thermal cycler (Bio-Rad) and the cycling was: an initial denaturation at $94^{\circ} \mathrm{C}$ for $30 \mathrm{sec}$, followed by 35 cycles of $7 \mathrm{sec}$ at $98^{\circ} \mathrm{C}$ and $2 \mathrm{~min}$ at $68^{\circ} \mathrm{C}$, and a final extension for $5 \mathrm{~min}$ at $72^{\circ} \mathrm{C}$. Three $\mu$ l of the PCR 

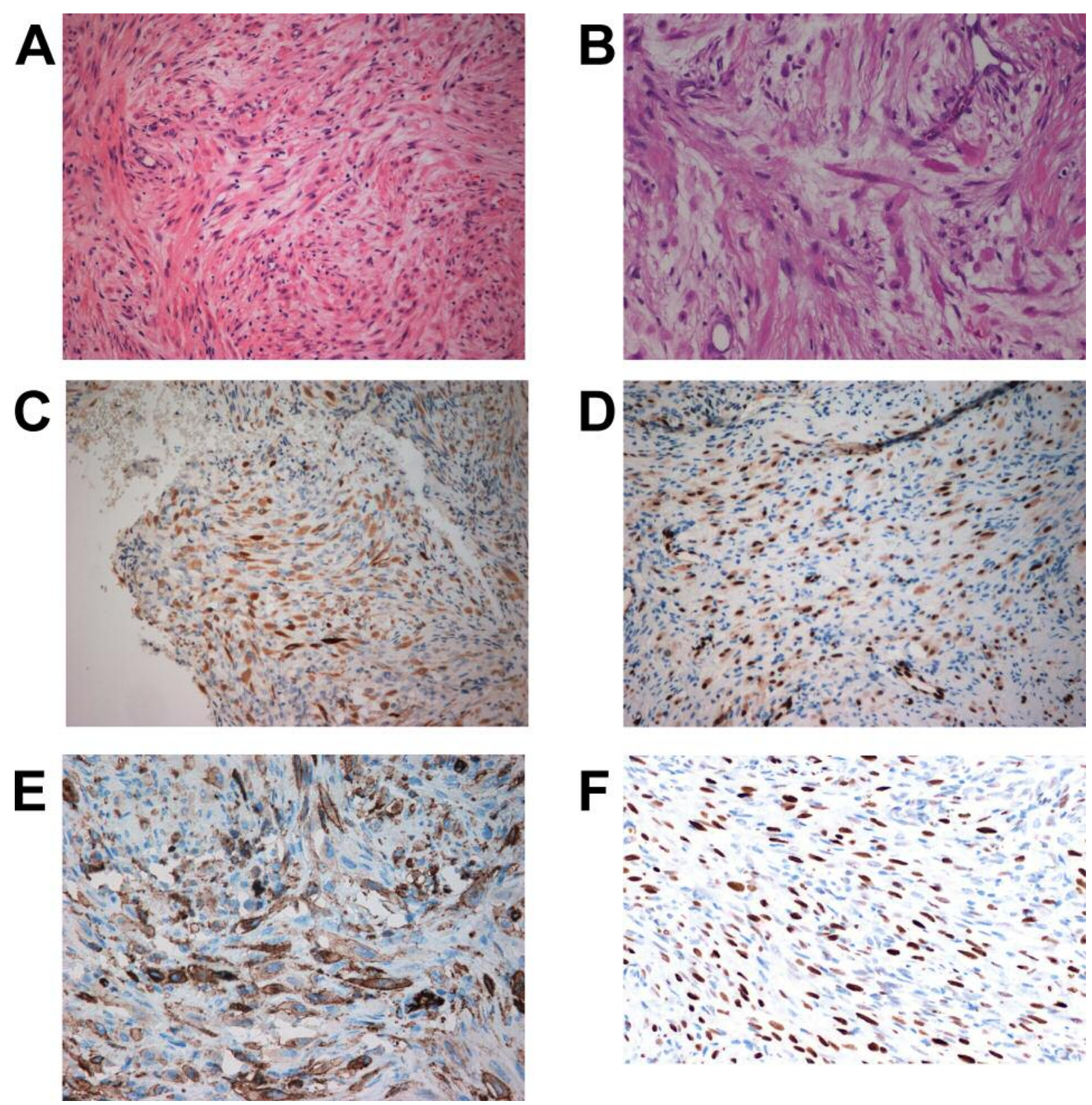

Figure 1. Microscopic examination of the pseudomyogenic hemangioendothelioma. A) H\&E-stained section showing a tumor with a spindle cell lesion without atypia, $\times 20 . B) H \& E$-stained section, $\times 40$. C) Immunoexpression of cytokeratin AE1/AE3, $\times 20 . D)$ Immunoexpression of ERG, $\times 20$, E) Immunoexpression of CD31, $\times 20 . F)$ Immunoexpression of FOSB showing strong nuclear positivity, $\times 20$.

products were stained with GelRed (Biotium, VWR International, Oslo, Norway), analyzed by electrophoresis through $1.0 \%$ agarose gel, and photographed. The remaining PCR products were purified using the MinElute PCR Purification Kit (Qiagen) and analysed by direct sequencing using the dideoxy procedure with the BigDye terminator v1.1 cycle sequencing kit (ThermoFisher Scientific, Waltman, MA, USA) on the Applied Biosystems Model 3500 Genetic Analyzer sequencing system. The BLAST software (http://blast.ncbi.nlm.nih.gov/Blast.cgi) was used for computer analysis of sequence data.

\section{Results}

The G-banding analysis revealed a normal karyotype 46, XX in all examined 25 metaphases (data not shown).

Using the "grep" command and a search term corresponding to the first $20 \mathrm{nt}$ in exon 2 of $F O S B$ on the raw RNA sequence data, which were in the text-based fastq format, 23 unique sequences were extracted (Table I). Alignment of each of them with the human genome using BLAT on the genome browser 


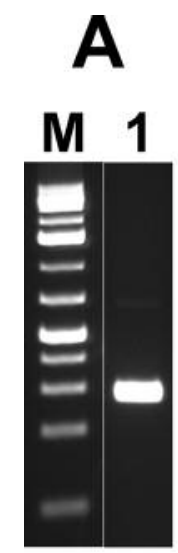

\section{B}

WWTR1 exon $4 \downarrow$ FOSB exon 2

C CAA GAG G A C T CA T G G GCA G GA T GC GC C G T T TC G G G GA

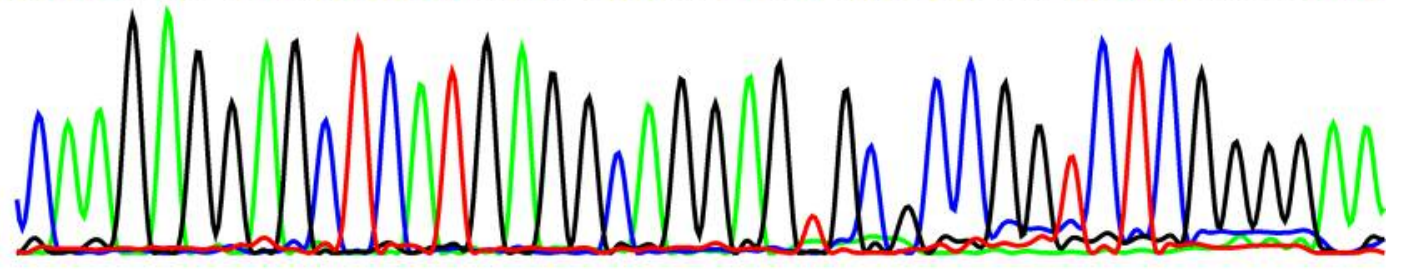

C

MNPASAPPPLPPPGQQVIHVTQDLDTDLEALFNSVMNPKPSSWRKKILPE SFFKEPDSGSHSRQSSTDSSGGHPGPRLAGGAQHVRSHSSPASLQLGTGA GAAGSPAQQHAHLRQQSYDVTDELPLPPGWFMTFTATGQRYFLNHIEKIT TWQDPRKAMNQPLNHMNLHPAVSSTPVPQRSMAVSQPNLVMNHQHQQQMA PSTLSQQNHPTQNPPAGLMSMPNALTTQQQQQQKLRLQRIQMERERIRMR QEELMR E CAGLGEMPGSFVPTVTAITTSQDLQWLVQPTLISSMAQSQGQ PLASQPPVVDPYDMPGTSYSTPGMSGYSSGGASGSGGPSTSGTTSGPGPA RPARARPRRPREETLTPEEEEKRRVRRERNKLAAAKCRNRRRELTDRLQA ETDQLEEEKAELESEIAELQKEKERLEFVLVAHKPGCKIPYEEGPGPGPL AEVRDLPGSAPAKEDGFSWLLPPPPPPPLPFQTSQDAPPNLTASLFTHSE VQVLGDPFPVVNPSYTSSFVLTCPEVSAFAGAQRTSGSDQPSDPLNSPSL LAL

Figure 2. RT-PCR and Sanger sequencing of the PCR products in pseudomyogenic hemangioendothelioma. A) Gel electrophoresis showing the amplified WWTR1-FOSB cDNA fragment. M, GeneRuler $1 \mathrm{~Kb}$ DNA ladder (Thermo Scientific). Lane 1, amplification using the forward primer WWTR1-996F1 and the reverse primer FOSB-817R1. B) Partial sequence chromatograms of the cDNA amplified fragment showing the fusion (arrow) of WWTR1 with FOSB. C) The putative WWTR1-FOSB fusion protein. The WW domain from WWTR1 is in green, the bZIP region from FOSB is in yellow, the WWTR1-FOSB junction is in red.

(https://genome-euro.ucsc.edu/index.html) and BLAST on the NCBI database (https://blast.ncbi.nlm.nih.gov/Blast.cgi ) showed that 19 sequences were from $F O S B$ whereas 4 sequences were hybrids containing sequences from exon 4 of WWTR1 (sequence with accession number NM_015472) and exon 2 of FOSB (sequence with accession number NM_006732) (Table I).

RT-PCR with the primer set WWTR1-996F1/FOSB817R2 amplified a cDNA fragment strongly suggesting the presence of a WWTRl-FOSB fusion transcript in the examined tumor (Figure 2A, lane 1). Sequencing of the amplified cDNA fragment showed that it consisted of a WWTR1-FOSB chimeric fragment in which exon 4 of WWTRI (nucleotide 1322 accession number NM_012330 version 3) was fused in-frame to exon 2 of FOSB (Figure 2B). Thus, taking into account the relevant reference sequences NM_015472/NP_056287 for WWTRl and NM_006732/NP_006723 for FOSB, the fusion WWTR1FOSB appears to code for a 553 amino acid residue chimeric WWTR1-FOSB protein consisting of the first 257 and the last 296 (position 43-338) amino acid residues of the WWTR1 and FOSB proteins, respectively (Figure 2C). 


\section{Discussion}

In the present study, a WWTR1-FOSB fusion gene was identified in a pseudomyogenic hemangioendothelioma, the third fusion gene to be detected in tumors of this type. One consequence of the fusion is that it places coding regions of the FOSB gene under the control of the WWTRl promoter. Replacement of the native $F O S B$ promoter with the promoter of the fusion partner has been a theme common to all reported $F O S B$ fusions described (4-6, 9). For example, in the first reported SERPINEI-FOSB fusion gene in pseudomyogenic hemangioendothelioma, the breakpoint in SERPINE1 was in the non-coding region of exon 1 . Therefore, SERPINE1 provided a strong promoter for the expression of its fusion partner, FOSB (4). Similarly, in the ZFP36-FOSB fusion gene, exon 1 of ZFP36 was fused inframe to exon 2 of $F O S B$ and contributed only eight amino acid residues to the ZFP36-FOSB chimeric protein (9). Thus, the role of $Z F P 36$ in the fusion with $F O S B$ was to provide a promoter for regulating the expression of $F O S B$.

In the present case, however, the fusion WWTR $1-F O S B$ appears to code for a chimeric transcription factor containing the N-terminal 14-3-3 binding region and the WW domain from WWTR1 $(10,11)$. It also contains the region required for transformation activity, the basic-leucine zipper (bZIP) domain, and the $\mathrm{C}$-terminal transcriptional activation domain from FOSB $(10,11)$. A WWTR 1-FOSB fusion gene was also reported previously, in an epithelioid hemangioma (9) where exon 3 of WWTRl was fused in-frame with exon 1 of $F O S B$, and the coding chimeric WWTR1-FOSB protein contained the above-mentioned functional regions.

The recently described $A C T B-F O S B$ fusion gene in pseudomyogenic hemangioendothelioma also codes for a chimeric transcription factor $(5,6)$. The ACTB-FOSB protein contains the nucleotide-binding domain of the sugar kinase/HSP70/actin superfamily from ACTB and all the above-mentioned regions of FOSB (12). However, there seemed to be no clinicopathological differences between pseudomyogenic hemangioendothelioma carrying an $A C T B$ $F O S B$ fusion and the tumors with SERPINE1-FOSB, except that $A C T B-F O S B$ positive tumors were often solitary (5).

At the genomic level, WWTRl (on chromosome band $3 \mathrm{q} 25)$ is transcribed in the direction from telomere to centromere whereas the transcription of FOSB (on chromosome band $19 \mathrm{q} 13$ ) proceeds in the opposite direction, from centromere to telomere. Hence, formation of a WWTR1-FOSB fusion should not be possible from a simple and balanced $\mathrm{t}(3 ; 19)(\mathrm{q} 25 ; \mathrm{q} 13)$ translocation. An additional genomic aberration would be required, for example an inversion or an insertion in one of the derivative chromosomes, der(3) and $\operatorname{der}(19)$. The orientation of $W W T R I$ relative to $F O S B$ may also explain the possibly low frequency of WWTRl-FOSB fusions. The SERPINE1-FOSB fusion gene is the result of a balanced $\mathrm{t}(7 ; 19)(\mathrm{q} 22 ; \mathrm{q} 13)$ chromosomal aberration because SERPINE1 and FOSB are transcribed in the direction from centromere to telomere (3, 4). The $A C T B-F O S B$ fusion gene could also be the result of a balanced $\mathrm{t}(7 ; 19)(\mathrm{p} 22 ; \mathrm{q} 13)$ since $A C T B$ on $7 \mathrm{p} 22$ is transcribed from centromere to telomere, although such a translocation has not been reported yet in pseudomyogenic hemangioendotheliomas $(5,6)$.

The SERPINE1-FOSB, ACTB-FOSB, and WWTR1-FOSB fusion genes and the taking over of FOSB expression control by SERPINE1, ACTB, and WWTRI strong promoters makes FOSB a useful immunohistochemical diagnostic marker for pseudomyogenic hemangioendotheliomas as it was the case here $(5,13,14)$. However, genomic analyses are needed to clarify which gene recombinations occur at which frequencies in these tumors, and also whether as yet unknown variants of the $F O S B$-activation theme exist.

\section{Conflicts of Interest}

The Authors declare that they have no potential conflicts of interest exist.

\section{Authors' Contributions}

IP designed the research, performed the molecular genetic analyses, interpreted the data, and wrote the manuscript. IL did the pathological evaluations. LG performed cytogenetic experiments and interpreted the data. SH evaluated the cytogenetics wrote the manuscript.

\section{Acknowledgements}

The Authors thank Professor G. Petur Nielsen, M.D., Department of Pathology, Massachusetts General Hospital, Boston for help with diagnosis, performing FOSB IHC analysis and providing Figure 1F. This work was supported by grants from Radiumhospitalets Legater.

\section{References}

1 Hornick JL and Fletcher CD: Pseudomyogenic hemangioendothelioma: a distinctive, often multicentric tumor with indolent behavior. Am J Surg Pathol 35: 190-201, 2011. PMID: 21263239. DOI: 10.1097/PAS.0b013e3181ff0901

2 Al-Qaderi A and Mansour AT: Pseudomyogenic Hemangioendothelioma. Arch Pathol Lab Med, 2018. PMID: 30576238. DOI: 10.5858/arpa.2017-0430-RS

3 Trombetta D, Magnusson L, von Steyern FV, Hornick JL, Fletcher CD and Mertens F: Translocation t(7;19)(q22;q13)-a recurrent chromosome aberration in pseudomyogenic hemangioendothelioma? Cancer Genet 204: 211-215, 2011. PMID: 21536240. DOI: 10.1016/j.cancergen.2011.01.002

4 Walther C, Tayebwa J, Lilljebjorn H, Magnusson L, Nilsson J, von Steyern FV, Ora I, Domanski HA, Fioretos T, Nord KH, Fletcher CD and Mertens F: A novel SERPINE1-FOSB fusion gene results in transcriptional up-regulation of $F O S B$ in pseudomyogenic haemangioendothelioma. J Pathol 232: 534540, 2014. PMID: 24374978. DOI: 10.1002/path.4322 
5 Agaram NP, Zhang L, Cotzia P and Antonescu CR: Expanding the spectrum of genetic alterations in pseudomyogenic hemangioendothelioma with recurrent Novel ACTB-FOSB gene fusions. Am J Surg Pathol 42: 1653-1661, 2018. PMID: 30256258. DOI: 10.1097/PAS.0000000000001147

6 Zhu G, Benayed R, Ho C, Mullaney K, Sukhadia P, Rios K, Berry R, Rubin BP, Nafa K, Wang L, Klimstra DS, Ladanyi M and Hameed MR: Diagnosis of known sarcoma fusions and novel fusion partners by targeted RNA sequencing with identification of a recurrent $A C T B-F O S B$ fusion in pseudomyogenic hemangioendothelioma. Mod Pathol, 2018. PMID: 30459475. DOI: 10.1038/s41379-018-0175-7

7 Panagopoulos I, Gorunova L, Bjerkehagen B and Heim S: The "grep" command but not FusionMap, FusionFinder or ChimeraScan captures the CIC-DUX4 fusion gene from whole transcriptome sequencing data on a small round cell tumor with t(4;19)(q35;q13). PLoS One 9: e99439, 2014. PMID: 24950227. DOI: 10.1371/journal.pone.0099439

8 Panagopoulos I, Gorunova L, Bjerkehagen B and Heim S: Novel $K A T 6 B-K A N S L 1$ fusion gene identified by RNA sequencing in retroperitoneal leiomyoma with $\mathrm{t}(10 ; 17)(\mathrm{q} 22 ; \mathrm{q} 21)$. PLoS One 10: e0117010, 2015. PMID: 25621995. DOI: 10.1371/ journal.pone. 0117010

9 Antonescu CR, Chen HW, Zhang L, Sung YS, Panicek D, Agaram NP, Dickson BC, Krausz T and Fletcher CD: ZFP36FOSB fusion defines a subset of epithelioid hemangioma with atypical features. Genes Chromosomes Cancer 53: 951-959, 2014. PMID: 25043949. DOI: $10.1002 /$ gcc.22206
10 Kanai F, Marignani PA, Sarbassova D, Yagi R, Hall RA, Donowitz M, Hisaminato A, Fujiwara T, Ito Y, Cantley LC and Yaffe MB: TAZ: a novel transcriptional co-activator regulated by interactions with 14-3-3 and PDZ domain proteins. EMBO J 19: 6778-6791, 2000. PMID: 11118213. DOI: $10.1093 / \mathrm{emboj} / 19.24 .6778$

11 Wisdom R and Verma IM: Proto-oncogene FosB: the amino terminus encodes a regulatory function required for transformation. Mol Cell Biol 13: 2635-2643, 1993. PMID: 8474434.

12 Hurley JH: The sugar kinase/heat shock protein 70/actin superfamily: implications of conserved structure for mechanism. Annu Rev Biophys Biomol Struct 25: 137-162, 1996. PMID: 8800467. DOI: 10.1146/annurev.bb.25.060196.001033

13 Hung YP, Fletcher CD and Hornick JL: FOSB is a Useful Diagnostic Marker for Pseudomyogenic Hemangioendothelioma. Am J Surg Pathol 41: 596-606, 2017. PMID: 28009608. DOI: 10.1097/PAS.0000000000000795

14 Sugita S, Hirano H, Kikuchi N, Kubo T, Asanuma H, Aoyama $\mathrm{T}$, Emori $\mathrm{M}$ and Hasegawa T: Diagnostic utility of FOSB immunohistochemistry in pseudomyogenic hemangioendothelioma and its histological mimics. Diagn Pathol 11: 75, 2016. PMID: 27515856. DOI: 10.1186/s13000-016-0530-2

Received April 6, 2019

Revised May 26, 2019

Accepted May 27, 2019 